\title{
Short-Term Variations of LBV's
}

\author{
Otmar Stahl
}

Landessternwarte Königstuhl, D-69117 Heidelberg, Germany

\section{Introduction}

LBV's are variable on many different time scales from weeks to years or even decades. The typical LBV variations on timescales of years or longer are covered in the paper by Humphreys (this volume). Here I discuss variations of LBV's on timescales from weeks to years.

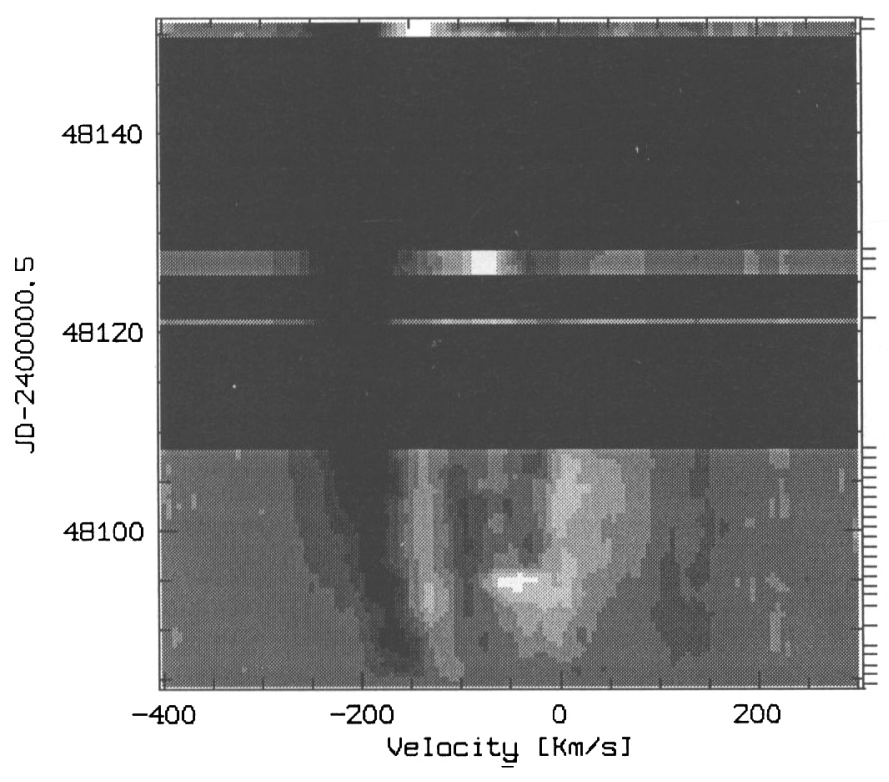

Fig. 1. Dynamical spectrum of P Cygni in the line FeIII $\lambda 5156$ obtained at the Landessternwarte Heidelberg in 1990 with FLASH. The mean spectrum has been subtracted to enhance the visibility of variable features. Note the outwards accelerating absorption feature.

Most classes of stars are little studied on these timescales. This also holds true for LBV's. Therefore the hot star group of the Landessternwarte Heidelberg started a monitoring program of a few bright LBV's (and other objects) 
using the $70 \mathrm{~cm}$-telescopes at the Landessternwarte and later the ESO $50 \mathrm{~cm}$ telescope at La Silla. For more details about the project see the paper by Kaufer (this volume).

\section{$2 \quad$ P Cygni}

The star P Cygni is the brightest LBV. It has been relatively stable in the last centuries and is classified as an LBV only because of its historic outburst in the 17th century. At present it shows a spectrum of a B1.5 supergiant. Most lines show P Cygni profiles.

Our observations show that $\mathrm{P}$ Cygni shows variations similar to other early-B supergiants (see paper by Wolf, this volume). The spectroscopic variability seems to be dominated by expanding and accelerating shells.

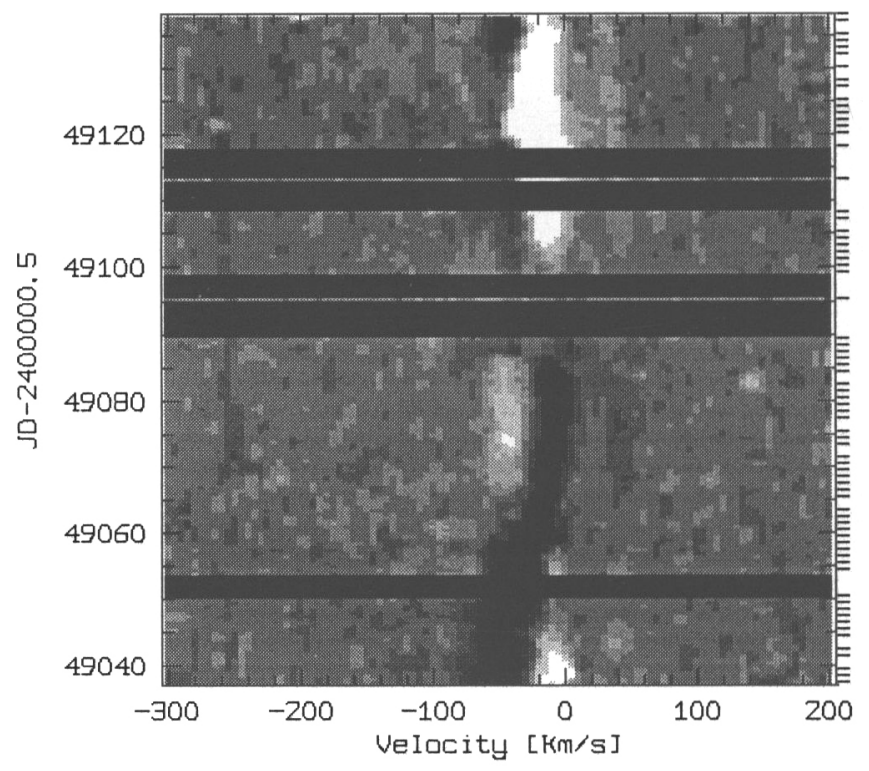

Fig. 2. Dynamical spectrum of HD 160529 around the line FeII $\lambda 6432$ obtained with the ESO 50cm-telescope at La Silla in 1993 with FLASH. Note the absortion feature moving towards longer wavelengths.

\section{$3 \quad$ HD 160529}

HD 160529 was discovered as an LBV by Sterken et al. (1991). It is a relatively cool LBV (late-B to early-A) with a small photometric amplitude $(\approx 0.5 \mathrm{mag})$ and a comaparatively low luminosity. 
The spectroscopic variations of HD 160529 are markedly different from those of P Cygni: Accelerating shells have never been observed in this object. In contrast, absorption features moving from red to blue in the line profiles are often observed. This can be regarded as evidence for rotational modulation, which is common in late-B and early-A supergiants (see paper by Kaufer, this volume).

\section{AG Car}

AG Car is the brightest of the active LBV's and also known for its nebula. It shows brightness variations from magnitude 8 to 6 on timescales of years. During the outburst, the spectral type varies between Ofpe/WN9 and earlyA. Its last outburst started around 1990.

We covered most of this outburst spectroscopically with observations typically spanning four months in each season. The behaviour over the full outburst cycle indicates an asymmetry between the ascending and the descending branch. The emission lines are much stronger after the maximum, possible indicating a higher luminosity.

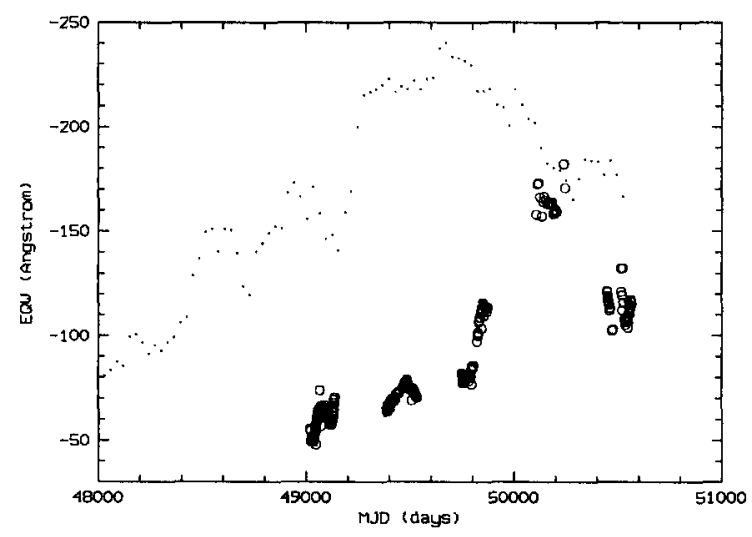

Fig. 3. The development of the equivalent width of $\mathrm{H} \alpha$ of $\mathrm{AG}$ Car during the recent outburst (large symbols). The data have been obtained with FLASH, Heros at the ESO 50cm-telescope. The visual lightcurve is overplotted in small symbols. Note that the maximum of the equivalent widths occurs much later than the visual maximum.

\section{Conclusions}

The short-term spectroscopic variations of LBV's in relative quiescence are qualitatively similar to variations shown by normal supergiants of similar 
spectral type. In particular, in LBV's of later spectral type and lower luminosity rotational modulation is more pronounced than in LBV's of higher temperature and luminosity. The timescales in LBV's are probably longer tahn in normal supergiants. This could be due to their higher luminosity.

The short-term variations of LBV's during outburst are very complex and indicate that the details of LBV outbursts are still not understood. The behaviour of AG Car during rise to visual maximum is markedly different from the decline to minimum. This may indicate that a simple change of radius and temperature does not explain LBV outbursts.

\section{Discussion}

M. Magalhães: Polarisation data of $\mathrm{P}$ Cygni show night-to-night variation of about $0.1 \%$. You have described longer-term variations, of weeks to months, in the photometric light curve of $\mathrm{P}$ Cygni. Could you comment on night-to-night variations in the photometric data of $\mathrm{P}$ Cygni?

O. Stahl: I am not aware of any significant night-to-night variations in photometric data. Polarisation seems to be the only quantity which shows these variations in $\mathrm{P}$ Cygni.

H. Lamers: You showed that AG Car displays its maximum $\mathrm{H} \alpha$ emission after the photometric maximum. Maybe the star was too cold during the outburst to keep the wind ionised and only after the outburst was the star hot enough to ionise the wind again?

O. Stahl: It is indeed possible that the star is too cool in outburst to keep the wind ionised. This does not, however, explain the asymmetry in the $\mathrm{H} \alpha$ emission with respect to the optical outburst. 